\title{
Norois
}

Environnement, aménagement, société

$247 \mid 2018$

Recherche touristique : perspectives latinoaméricaines

\section{Inventando el mundo maya}

Los inicios de la aviación y el turismo en Yucatán, 1929-1969

Inventer le monde maya. Les débuts de l'aviation et du tourisme au Yucatan, 1929-1969

Inventing the Mayan World. The Beginnings of Aviation and Tourism in Yucatán, 1929-1969

\section{Elda Moreno Acevedo}

\section{OpenEdition}

\section{Journals}

\section{Edición electrónica}

URL: http://journals.openedition.org/norois/6733

DOI: $10.4000 /$ norois. 6733

ISBN: 978-2-7535-7629-2

ISSN: $1760-8546$

\section{Editor}

Presses universitaires de Rennes

\section{Edición impresa}

Fecha de publicación: 19 septiembre 2018

Paginación: 95-106

ISBN: 978-2-7535-7570-7

ISSN: 0029-182X

\section{Referencia electrónica}

Elda Moreno Acevedo, «Inventando el mundo maya », Norois [En ligne], 247 | 2018, mis en ligne le 19 septembre 2020, consulté le 04 janvier 2021. URL : http://journals.openedition.org/norois/6733 ; DOI : https://doi.org/10.4000/norois.6733 


Presses
Universitaires
de Rennes
www.pur-editions.fr
Revue en ligne : http://norois.revues.org

\title{
Nota de investigación Inventando el mundo maya Los inicios de la aviación y el turismo en Yucatán, 1929-1969
}

\author{
Note de recherche - Inventer le monde maya \\ Les débuts de l'aviation et du tourisme au Yucatan, 1929-1969 \\ Research note - Inventing the Mayan World \\ The Beginnings of Aviation and Tourism in Yucatán, 1929-1969
}

\section{Elda Moreno Acevedo}

Facultad de Ciencias Antropológicas (Universidad de Yucatán) - Km 1 carretera Mérida-Tizimín, Cholul, CP 97305,

Mérida, Yucatán, México. (macevedo@correo.uady.mx)

Resumen: Este artículo cuestiona el rol del surgimiento de la aviación comercial en el inicio del turismo en la península de Yucatán durante las primeras décadas del siglo XX. Se propone que ambos procesos se encuentran vinculados y coinciden con la "invención del mundo maya”. Al conocerse y difundirse los múltiples asentamientos mayas de la región inició el proceso de invención de un mundo mítico que en esos años fue objeto de interés arqueológico internacional y, posteriormente, con el inicio de la conectividad aérea y la posibilidad de llegar a los destinos insulares del Caribe yucateco, convirtieron a Yucatán en un atractivo turístico. Es importante señalar que la península, debido a su condición geográfica estuvo históricamente confinada y aislada del resto de la República Mexicana. Por tanto, el surgimiento y consolidación del transporte aéreo y el interés por conocer el mundo maya tuvo amplias repercusiones en el desarrollo turístico de la región.

Résumé : Cet article vise à interroger le rôle du développement de l'aviation commerciale dans l'apparition du tourisme dans la péninsule du Yucatan au cours des premières décennies $d u$ Xx siècle. L'hypothèse formulée suggère l'existence de liens étroits entre ces deux processus cö̈ncidant avec "l'invention du Monde Maya». En donnant à connaître la présence de nombreux sites mayas dans la région, ce phénomène a initié un processus d'invention d'un monde mythique faisant l'objet d'un intérêt archéologique à l'échelle internationale, tandis que le développement de la connectivité aérienne a offert la possibilité d'accéder de nouvelles destinations insulaires de la Caraïbe yucatèque. De cette manière, l'apparition et la consolidation du transport aérien et l'intérêt pour le monde maya ont eu d'importantes répercutions sur le développement touristique de cette région marquée par un isolement historique par rapport au reste du pays.

Abstract: The contribution seeks to explain the confluence of two processes: the beginning of commercial aviation, and the emergence of tourism in the Yucatán peninsula during the first decades of the 20th century. Both processes are closely linked to the invention of the Mayan world. As the Maya's multiple settlements became known and this knowledge spread, so began the process of inventing a mythical world that, at this time, was the subject of international archaeological interest and, later, with the development of aerial connectivity, became a tourist attraction. The emergence and consolidation of air transport and the interest in the Mayan world had wide-reaching repercussions on the development of the region, whose geographical position was historically confined and isolated from the rest of Mexico. 
Palabras clave: aviación comercial - turismo - Mayas - Yucatán

Mots clés : aviation commerciale - tourisme - Mayas - Yucatan

Keywords: commercial aviation - tourism - Maya - Yucatán

\section{INTRODUCCIÓN}

En octubre de 1929, Charles A. Lindbergh sobrevoló la península de Yucatán en un aeroplano Sikorsky. El afamado aviador, como parte de una expedición de «reconocimiento científico» auspiciada por la Pan American Airways Inc. y The Carnegie Institution tomó fotografías panorámicas de la entonces denominada Área Maya que dieron la vuelta al mundo y mostraron a propios y extraños, por primera vez, las imágenes completas de ciudades prehispánicas, vestigios arqueológicos y atractivos naturales que permanecían sin explorar, ocultos por la densa vegetación de la selva ${ }^{1}$.

Si bien desde la primera mitad del siglo XIX ya se conocía la magnificencia de la civilización maya por las narraciones e ilustraciones de viajeros y exploradores, en un primer momento esta información se concentró en ciertos círculos internacionales formados por estudiosos, curiosos y excéntricos. Fue hasta la segunda década del siglo XX que la mitología sobre la civilización maya comenzó a formarse y difundirse por iniciativa norteamericana (Palacios, 2012), y dio inicio el proceso de invención y difusión de lo que ahora se conoce mundialmente como el «mundo maya». La coyuntura fue resultado de la confluencia de procesos políticos, sociales y arqueológicos que coincidieron con un cambio tecnológico crucial, el avión. Así, para la península de Yucatán, los inicios del turismo están estrechamente ligados con el surgimiento de la aviación comercial y la invención del «mundo maya». Este último proceso lo identificamos a partir de los planteamientos de Boyer (2002) que señala cómo los atractivos y las prácticas turísticas surgen como un esfuerzo de

1. Los resultados y fotografías de dicha expedición fueron publicados en The Geographical Review de abril de 1930. La expedición fue parte de una estrategia comercial de la Pan American Airways que en ese año concretó la compra de las acciones de la Compañía Mexicana de Aviación (CMA), con lo que dio inicio a sus rutas internacionales. Charles A. Lindbergh comandó vuelos, diseñó rutas y colaboró en la estructuración de la empresa. [http://mx.reuters.com/article/topNews/idMXN27102256 20100827 ?pageNumber $=3 \&$ virtualBrandChannel $=0 \&$ sp $=$ true $]$.

distinción por un sector de la sociedad y se difunden a diversos ritmos entre los grupos sociales.

El turismo, como actividad humana con importantes repercusiones económicas, a partir de 1970 gradualmente adquirió una naturaleza masiva vinculada con el surgimiento y consolidación de la aviación comercial. Sin embargo, poco se sabe sobre la historia entrelazada de ambas actividades. Los estudios especializados sobre el turismo dedican breves párrafos a la historia de la aviación como mero detalle anecdótico, centrando su atención en las cifras del transporte aéreo en años recientes (Coltman, 1998; Page y Connell, 2006) o en el desarrollo tecnológico de los aviones para la industria militar (Carranza, 1976; Olsen, 2010). Este artículo tiene como objetivo principal mostrar el rol de la aviación comercial en los inicios del turismo durante la primera mitad del siglo XX en la Península de Yucatán, una región cuya condición geográfica la mantuvo históricamente confinada y aislada del resto de la república mexicana al menos hasta 1950, cuando la vía férrea abierta llegó a tierras yucatecas (García, 2006). La comunicación terrestre por medio de carreteras fue más tardía, pues la carretera federal 180, también conocida como Circuito del Golfo o Costera del Golfo fue construida por tramos desde 1954 hasta su conclusión en la década de 1960 (Banobras, 1994). Por tanto, el surgimiento y consolidación del transporte aéreo comercial en Yucatán durante el siglo XX tuvo amplias repercusiones sociales y económicas para la región peninsular y sobre todo en el ámbito turístico.

\section{LOS INICIOS DE LA AVIACIÓN EN YUCATÁN, I 9 I I - I 927}

En México, la aviación inició en enero de 1910 cuando el joven Alberto Braniff a bordo de un aeroplano Voissin, de manufactura francesa, surcó los cielos de la entonces hacienda de Balbuena (Buergo, Morlett, 2008). Un año después inició la aviación yucateca, el pionero fue Francisco Montalvo Castro, 
quien al decir de Rosado Espínola (1981; 1979) en aquellas épocas ejercía como boticario en el barrio de Santa Ana en Mérida y, motivado por su afición a la aviación mandó construir un biplano de madera, el cual se elevó sobre Paseo de Montejo piloteado por Gustavo Castaldi. La hazaña terminó en un aterrizaje donde el piloto salió ileso.

Para 1912 el aeroplano ya era una maquinaria con mayor difusión y comenzó a ser vista como un signo de modernidad con múltiples usos recreativos y prácticos. En Yucatán durante esa época se vivió un ambiente de prosperidad y riqueza en manos de un selecto grupo de familias que controlaban la millonaria industria del henequén (Quezada, 2001). Como señalan Pérez de Sarmiento y Savarino (2001), «esos años, conocidos como la belle époque trajeron un número asombroso de novedades a México y Yucatán, tales como el telégrafo, el teléfono, el fonógrafo, el cinematógrafo, la luz eléctrica y el automóvil». Parte importante de estas novedades fueron los aeroplanos, que pronto comenzaron a publicitarse en Mérida y fueron bien recibidos por los empresarios y comerciantes yucatecos, que ávidos de la modernidad acogían la nueva tecnología como parte del progreso. Así, Fernando Barbachano Bolio, convencido de que el aeroplano era una excelente opción para publicitar sus negocios, contrató a dos aviadores para que efectuaran una exhibición aérea. El evento se realizó en el puerto de Progreso y se organizó en forma de un espectáculo de circo que para esos años registraba su mayor apogeo (Pérez de Sarmiento y Savarino, 2001). El gran día causó mucha expectación entre los yucatecos. Dos trenes repletos de gente llegaron al lugar donde multitud de curiosos de todas las edades, atraídos por la banda musical y la algarabía "compraron boletos y presenciaron las piruetas aéreas”. (Rosado, 1979).

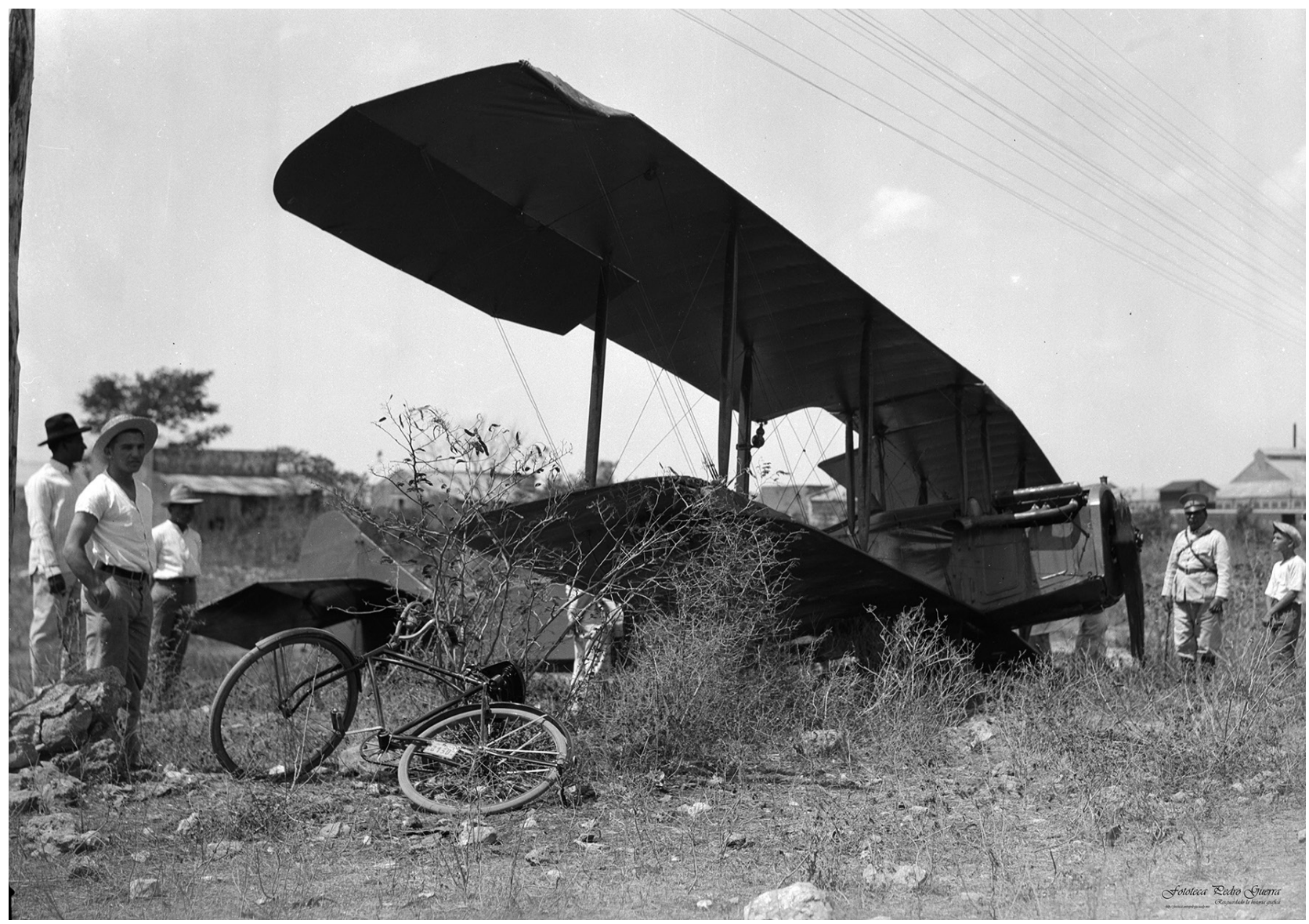

Figura 1: Avión Biplano aterriza en el campo yucateco (Fototeca Pedro Guerra)

Avion biplan au sol, quelque part au Yucatan (photothèque Pedro Guerra)

Biplane landing in the Yucatecan countryside (source: Fototeca Pedro Guerra) 


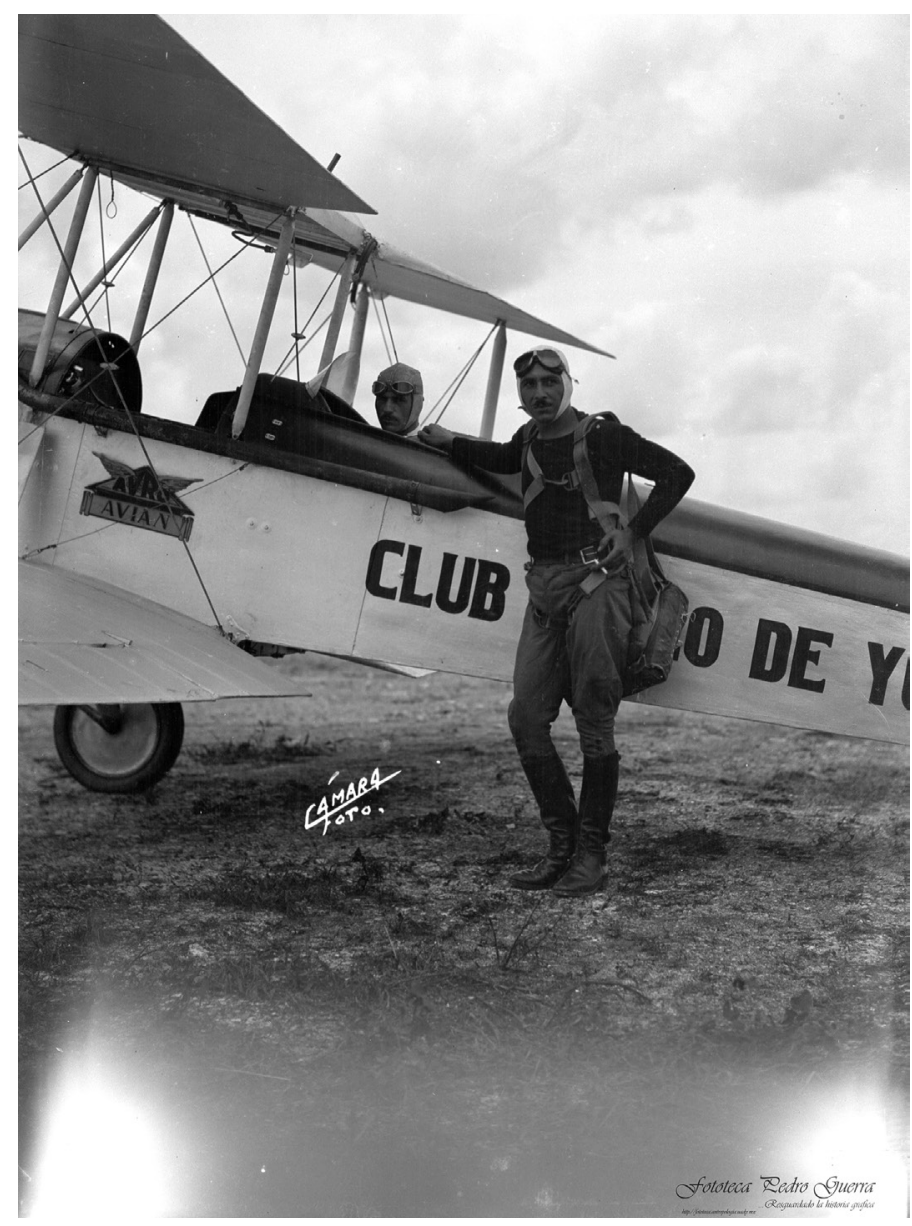

Figura 2: El osado aviador llamado "El Loco del Aire" (Fototeca Pedro Guerra) Pedro Guerra)

Le courageux aviateur surnommé "le Fou de l'air» (photothèque Pedro Guerra)

The daring aviator known as "The Madman of the Air" (Fototeca

Los cambios políticos también influyeron en el desarrollo de la aviación en México. El presidente Francisco I. Madero, en noviembre de 1911, se convirtió en el primer presidente de un país que utilizó una aeronave. Unos años después, en febrero de 1915 el presidente Venustiano Carranza decretó la creación de la Flotilla Aérea Constitucionalista, que después se convirtió en la Fuerza Aérea Mexicana². La primera misión de este escuadrón aéreo militar fue apoyar la campaña de Salvador Alvarado con demostraciones aéreas y volantes (Rosado, 1979). Los pobladores de Hecelchakán, Maxcanú, Tixkokob, Motul y Mérida reaccionaron con asombro ante esta nueva forma de propaganda política, en

2. [http://www.sedena.gob.mx/index.php/conoce-la-sedena/antecedenteshistoricos/fuerza-aerea-mexicana]. la cual no sólo se hizo publicidad, sino también se exhibió el avión como una maquinaria que simbolizaba el poderío y la modernidad del nuevo gobierno.

Con la creación del primer motor a reacción a finales de la década de 1920 la aviación comenzó a perfilarse como una alternativa de transporte, pues fue posible ampliar el tamaño de las aeronaves y realizar vuelos transcontinentales. En México, los inicios de la aviación comercial se remontan a 1920, cuando el descubrimiento de los pozos petroleros entre San Luis Potosí y Tamaulipas detonó el interés de los empresarios estadounidenses Lloyd A. Winship, Harry J. Lawson y Elmer C. Hammond, quienes fundaron la Compañía Mexicana de Transportación Aérea (CMTA) en julio de 1921 y, recibieron la primera concesión del gobierno mexicano para operar una ruta aérea: México-Tampico ${ }^{3}$.

A partir de 1921 diversas ciudades como Veracruz y Tampico comenzaron a ser vistas como puntos de destino en México para las rutas aéreas comerciales. En esos años la industria henequenera yucateca estaba en pleno declive con la caída del precio de la fibra al concluir la Primera Guerra Mundial. Sin embargo, en Mérida la clase empresarial local todavía contaba con recursos, estaba sujeta a préstamos y a líneas de crédito de los bancos neoyorquinos y, por lo tanto, la ciudad y el puerto de Progreso todavía eran puntos neurálgicos de los intereses comerciales internacionales (Lapointe, 2008). Hacia 1925, dos empresas estaban interesadas en iniciar operaciones aéreas en Yucatán. La Sociedad Colombo-Alemana de Transporte Aéreo (SCDTA, actualmente AVIANCA) propuso la ruta de Progreso a los principales destinos del Golfo (Veracruz y Tamaulipas) y creó un enlace entre Progreso y Frontera (Tabasco) ${ }^{4}$. Por su parte la Deutscher Aero Lloyd propuso la ruta Veracruz-Progreso. Esta última inició operaciones en 1927 con un hidroavión que hizo escalas en Villahermosa, Ciudad del Carmen y Campeche. La ruta fue cancelada días después, pues el hidroavión sufrió un accidente (Rosado, 1979). La desaparición de la ruta canceló momentáneamente el afán de comunicar Yucatán con el centro del país.

\footnotetext{
3. [http://haviacionmexicana.blogspot.mx/search $\% 5 \mathrm{D}, \% 5 \mathrm{Bhttp}: / / \mathrm{mx}$. reuters. com/article/topNews].

4. [http://scadta.co/colombo-alemana/].
} 


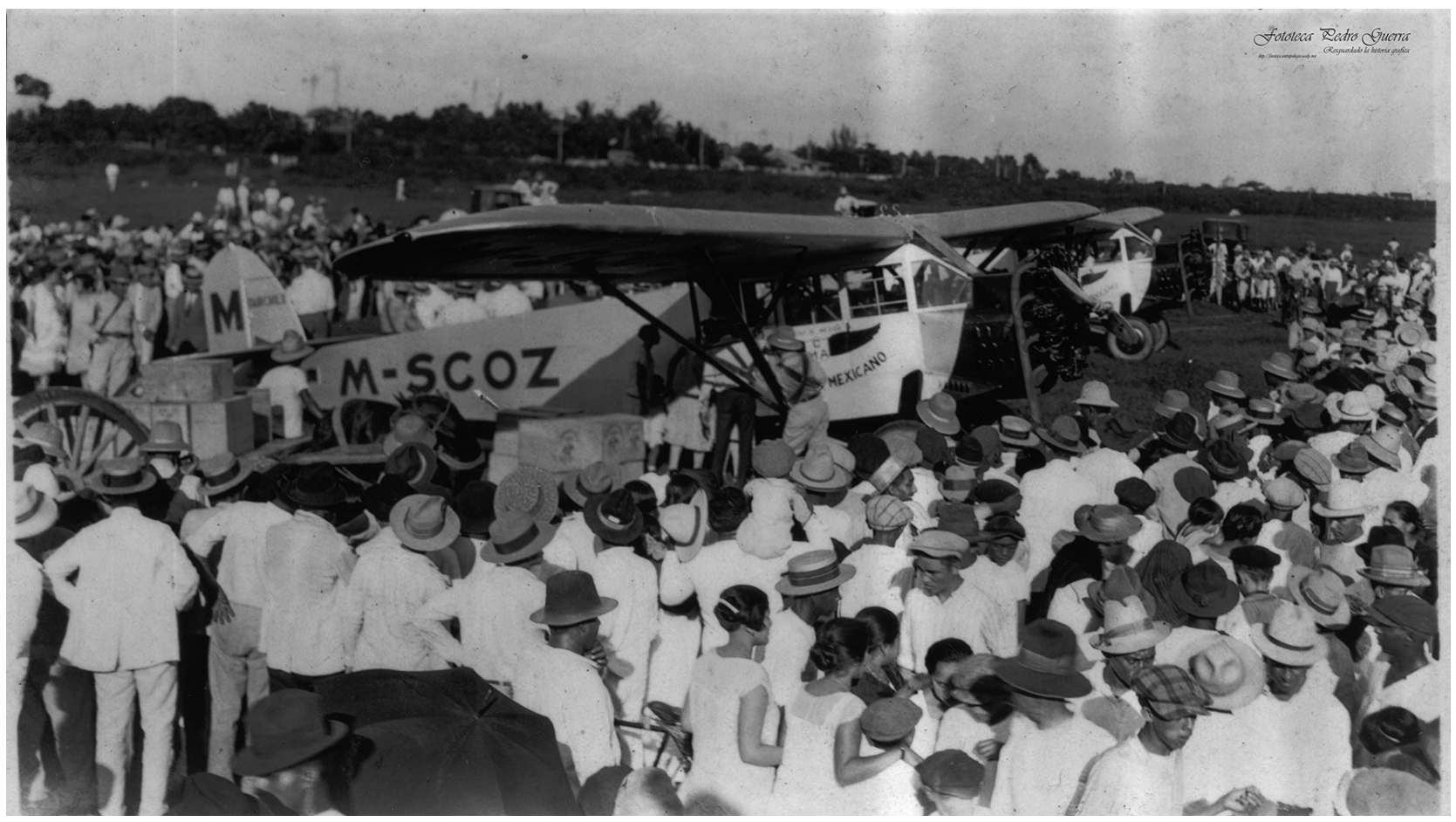

Figura 3: La multitud de curiosos observa los aviones (Fototeca Pedro Guerra) La foule de curieux devant les avions (photothèque Pedro Guerra) A crowd of onlookers observes the airplanes (source: Fototeca Pedro Guerra)

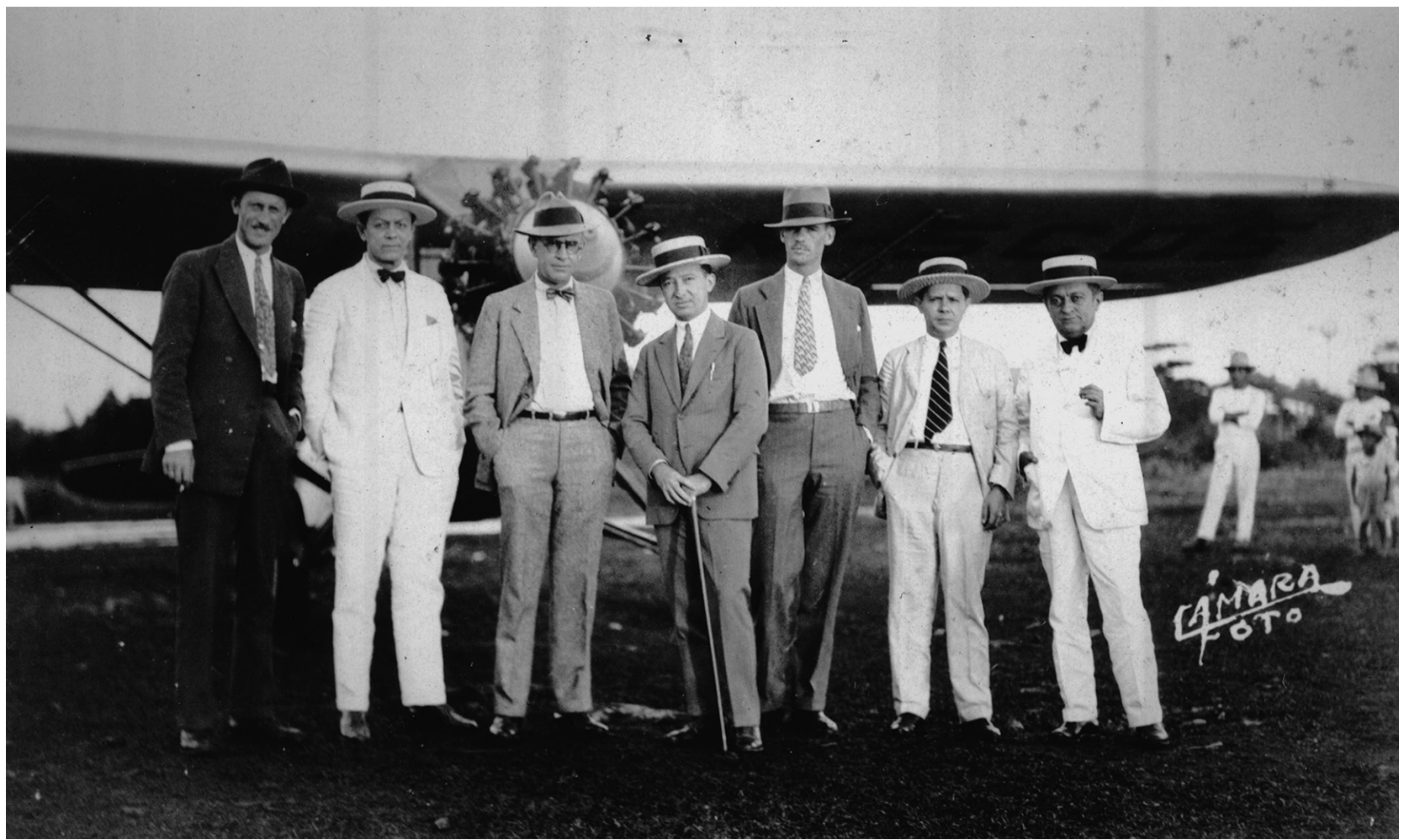

Figura 4: Hombres de negocios yucatecos fotografíados junto a un avión (Fototeca Pedro Guerra) Des hommes d'affaires yucatèques près d'un avion (photothèque Pedro Guerra) Yucatecan businessmen photographed alongside an airplane (Fototeca Pedro Guerra) 


\section{LA INVENCIÓN Y DIFUSIÓN DEL MUNDO MAYA, I923-I940}

El proceso de invención de la mitología sobre la civilización maya inició en el siglo XIX con los testimonios de viajeros y exploradores como Stephens, Catherwood y Charnay. Su difusión a gran escala comenzó en Estados Unidos en 1923. The New York Times publicó en marzo una nota sobre el tesoro rescatado en el fondo del cenote sagrado de Chichén Itzá conformado por piezas invaluables como máscaras de jade, ornamentos de oro, turquesas y un sinfín de objetos que permanecían en resguardo en el Museo Peabody (Palacios, 2012). Ese mismo año la denuncia pública de que dichos objetos fueron extraídos del cenote y trasladados a Estados Unidos dio pie a un gran escándalo internacional que estalló en torno a las exploraciones en Chichen Itzá realizadas por el entonces cónsul de Estados Unidos en Yucatán (Palacios, 2012; Pool, 2014). El interés sobre las ciudades mayas fue creciente y en 1923 se dio a conocer un contrato con vigencia de diez años entre The Carnegie Institution y la Dirección de Agricultura y Fomento de México, la cual tenía bajo su responsabilidad el departamento de Arqueología. Dicho documento autorizaba la explotación y restauración de los monumentos arqueológicos de Chichén Itzá y también dejaba abierta la posibilidad de explorar toda la península de Yucatán (Barrera, 2015).

Con esta medida Yucatán se volvió parte de los destinos de exploración del grupo de "arqueología científica" auspiciado por The Carnegie Institution (Palacios, 2015), cuyos investigadores y representantes comenzaron a referirse al conjunto de los sitios prehispánicos ubicados en Honduras, Belice, El Salvador, Guatemala y en el sureste de México como el "Área maya". Es en esta coyuntura que las exploraciones aéreas de Charles Lindbergh en 1926 y las primeras fotografías de las ciudades mayas, que muestran también la belleza de la exótica selva peninsular generaron curiosidad en el ámbito internacional y comenzaron a perfilar a la civilización maya y en especial a la Península de Yucatán como un atractivo turístico.

Palacios (2012) postula que la construcción epistemológica del "Área maya" se concretó en la década de 1920 y fue auspiciada por una élite de anticuarios, coleccionistas, promotores científicos, empresarios y académicos norteamericanos que gravitaban alrededor de la ciudad de Boston. Por tanto el proceso de difusión del mundo maya inició en la segunda década del siglo XX. En un primer momento consistió en la divulgación de los hallazgos y la circulación en medios académicos y prensa, generando expectación y curiosidad en la población norteamericana sobre el Área maya, específicamente sobre la Penísula de Yucatán. La obra de Sylvanus G. Morley, quizá el arqueólogo más reconocido relacionado con las primeras incursiones en Chichén Itzá, contribuyó al proceso de difusión. Sus trabajos fueron pioneros en los estudios y las interpretaciones publicadas acerca de la sociedad maya y constituyeron, junto con los trabajos de Vaillant, Tozzer, Thompson y Brainerd el primer referente de conocimiento y discusión arqueológica. (Pool, 2014)

La idea de los mayas como un atractivo turístico se posicionó gradualmente en la sociedad norteamericana y después en México y Yucatán. La creación del Museo Arqueológico e Histórico de Yucatán en 1925 fue una muestra de un naciente proceso en donde comenzó a revalorarse el pasado y a distinguir la importancia de los vestigios arqueológicos como un patrimonio nacional (Barrera, 2015). El turismo, como actividad económica era incipiente y los viajeros de esos años aún no se denominaban turistas ${ }^{5}$. La coyuntura principal que marcó el ingreso definitivo de la península de Yucatán a la lista de los destinos turísticos internacionales fue el inicio de operaciones en Mérida de la aerolínea Pan Am en diciembre de 1931.

\section{EL NACIMIENTO DE LA AVIACIÓN COMERCIAL, I928-I945}

1928 se considera un año crucial en el desarrollo de la aviación comercial en México. El presidente Plutarco Elías Calles (1924-1928) firmó el Convenio Iberoamericano de Navegación Aérea y el Convenio sobre aviación comercial y en septiembre inició las obras del Puerto Aéreo Central, hoy Aeropuerto Internacional de la ciudad de México. De igual forma, se concedieron permisos para 13 rutas aéreas (Jiménez, 1993) y, se reconocieron diez localidades como puertos aéreos de servicio internacional, entre

\footnotetext{
5. Hacia finales del siglo XIX y principios del XX los documentos que mencionan a los viajeros que llegan a Yucatán usan los términos visitantes, excursionistas, exploradores o extranjeros (Palacios, 2015)
} 
ellos Progreso y Cozumel. Ese mismo año Yucatán entró de lleno a la aviación comercial, cuando la Compañía Mexicana de Aviación (CMA), como parte de su proceso de expansión inauguró el 15 de octubre la ruta Mérida-Veracruz (Rosado, 1981).

La segunda mitad de la década de 1930 está signada por el paulatino crecimiento de la aviación como transporte. De Mérida salían vuelos diarios con destino a la ciudad de México. El incremento en la demanda de vuelos propició el crecimiento del transporte aéreo de carga y que las empresas dedicadas a este servicio comenzaran a transportar pasajeros en distancias cortas (Rosado, 1981; Rico, 2001).

En el ámbito internacional, la empresa pionera que operó con éxito en la península de Yucatán fue la Pan American World Airways (Pan Am). El 4 de diciembre de 1931 inauguró la ruta Miami-La Habana-Mérida-Chetumal-Belice-Puerto Barrios (Guatemala) - San Salvador, que pronto adquirió notoriedad y gradualmente colocó a Yucatán dentro del circuito centroamericano que tuvo gran auge a partir de la década de 1940. La influencia de la aerolínea Pan Am para la inserción de Yucatán a una serie de rutas turísticas y destinos de negocios fue evidente, si tomamos en cuenta que fue esta empresa la que en 1946 inició la ruta MéridaNueva Orleans-Guatemala-Managua (Nicaragua) -Balboa (Panamá). En 1961 Pan Am inició la ruta Miami-Mérida y la ruta Miami-Tampico-MéridaMéxico, D.F (Rosado, 1981).

Es importante mencionar que Pan Am a partir de 1929 se convirtió en la empresa determinante de la historia del transporte aéreo mexicano, pues compró el total de las acciones de la Compañía Mexicana de Aviación S.A. (CMA) lo cual favoreció su crecimiento y su internacionalización. Hacia 1945, después de varias fusiones y adquisiciones Pan Am poseía el $45 \%$ y el $40 \%$ de las acciones de las compañías Mexicana de Aviación y de Aeronaves de México, las dos empresas pilares del transporte aéreo mexicano (Jiménez, 1993).

\section{Aeromaya, la primera aerolínea DE YUCATÁN}

En la década de 1960 se consolidaron las empresas aéreas internacionales que tuvieron como objetivo la aviación comercial. Yucatán no estuvo exento de esas iniciativas. El 1 de noviembre de 1966, en el Hotel Panamericana de Mérida fue inaugurada la oficina central de Aeromaya, la primera compañía aérea yucateca, la cual tuvo su origen en el afán de ofrecer una experiencia completa por parte de la empresa Barbachano Travel Service, que para ese año ya se encontraba consolidada y brindaba servicios turísticos (tours) operando la cadena de hoteles Mayaland, conocidos en esa época como los más emblemáticos en la península de Yucatán por su ubicación en las inmediaciones de las zonas arqueológicas de Chichén Itzá y Uxmal (La Jornada, 27-052007). La empresa aérea quedó en manos de siete yucatecos, encabezados por Fernando Barbachano Gómez Rul y en abril de 1969 su planta laboral constaba de 60 empleados ${ }^{6 .}$

Para esos años transportarse en avión de Mérida a la ciudad de México era, como señala Sergio Quezada (2001) "un lujo distante de los bolsillos del común”, por tanto, el surgimiento de una aerolínea que diera este servicio a precios accesibles y con salidas frecuentes impulsó la integración geográfica de Yucatán con el centro del país. Así, en noviembre de 1966 despegó del aeropuerto meridano el primer vuelo de Aeromaya, que cubrió la ruta Mérida-Tuxpan-ciudad de México. La demanda de pasajeros no se hizo esperar, seis días después Aeromaya duplicó los vuelos a la ciudad de México y el 10 de noviembre duplicó también sus vuelos de la ruta Mérida-Isla Mujeres-Cozumel-Chichen Itzá (Rosado, 1981).

En el ámbito nacional, las aerolíneas más importantes como la CMA y Aeronaves de México competían adquiriendo aviones con equipamiento cada vez más sofisticado. Este fenómeno también se extendió a Yucatán, pues Aeromaya comenzó a adquirir más aviones con tecnología de punta. En 1967 inauguró vuelos en la ruta Mérida-PalenqueTuxtla-Acapulco-Manzanillo-Guadalajara. En julio del año siguiente inauguró el circuito México, D.F.-Campeche-Mérida con un vuelo cuatro días de la semana. Aeromaya también comenzó a operar vuelos cortos con destino a Villahermosa, Ciudad del Carmen, Campeche y Chetumal, así como la famosa ruta directa Mérida-Cozumel. Como bien señala Rosado (1981), Aeromaya fue una empresa dinámica pues sólo le tomó un año posicionarse en el mercado aéreo y, pronto expandió su radio de

6. [http://www.flightglobal.com/pdfarchive/view/1969/1969-0623.html]. 


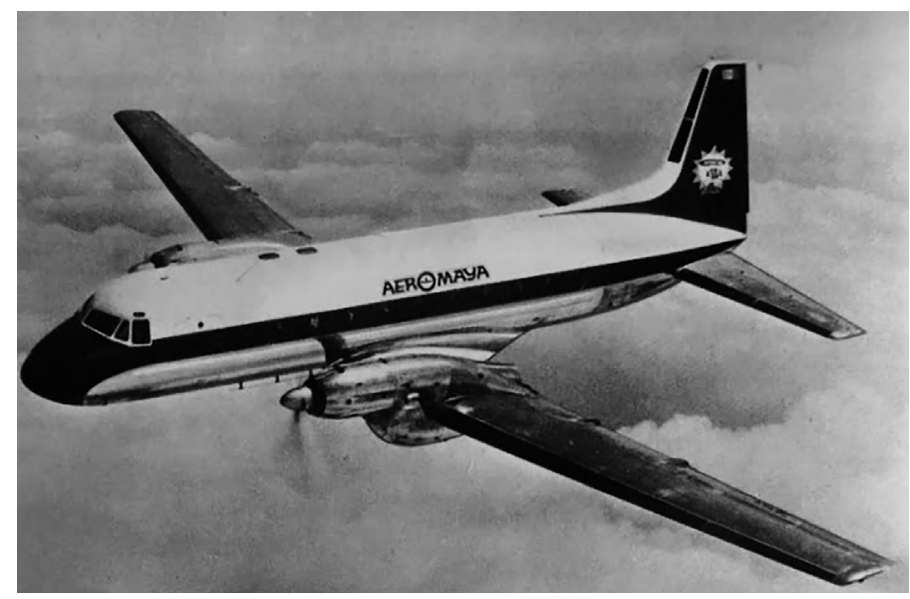

Figura 5: Un avión de Aeromaya, imagen utilizada probablemente como anuncio publicitario. [http://vamonosalbable.blogspot.mx/2014/01/aeromaya-una-de-las-pioneras-en-el.html]

Un avion de la Aeromaya - image probablement utilisée comme annonce publicitaire [http://vamonosalbable.blogspot.mx/2014/01/aeromaya-una-de-las-pioneras-en-el.html]

An Aeromaya aircraft; image probably used for advertising purposes (source: http://vamonosalbable.blogspot.mx/2014/01/aeromaya-una-de-laspioneras-en-el.html)

acción comunicando Mérida con las ciudades cercanas de la península, el sureste del país y los destinos estratégicos del territorio mexicano.

Una explicación plausible de este acelerado crecimiento es que Aeromaya fue fruto de las experiencias previas de Fernando Barbachano Gómez Rul y de un conjunto de empresarios yucatecos que operaron servicios aéreos en forma particular. De este modo, en el pasado de Aeromaya se pueden distinguir cuatro empresas aéreas: Aerotaxis, Aerosafari, Servicios Aéreos Gómez Méndez y Aerolíneas Vega las cuales, al fusionarse, contribuyeron a que la empresa aérea de Fernando Barbachano Gómez Rul reuniera los tres requisitos que según la teoría de los sistemas de transporte -infraestructura, flota de vehículos, operatividad y conocimiento empresarial del área-, la convirtieron no sólo en una empresa aérea, sino en una aerolínea que se erigió como un sistema de transporte eficaz y asequible para los estándares de la época (Izquierdo, 1994).

\section{Aeromaya, la historia de UnA AEROLINEA ORIENTADA AL TURISMO}

El antecedente de Aeromaya fue la empresa Aerotaxis, fundada en 1963 por Fernando Barbachano Ponce. Poco se sabe sobre los detalles operativos de la empresa, pero es posible suponer que tuvo corta vida. Dos años después, Fernando Barbachano Gómez Rul inauguró Aerosafari, empresa aérea que se dirigió a destinos de Quintana Roo como Isla Mujeres y Cozumel ${ }^{7}$. La crónica de Luis A. Ramírez Aznar en el Diario del Sureste del lunes 1 de noviembre de 1965 mencionó que Aerosafari "puso al servicio del turismo de todas las latitudes la bella ruta del Caribe que unirá todos los días a Mérida con Isla Mujeres y Cozumel [...] el DC-3 hizo en una hora y media el viaje hasta su primera estación, - Isla Mujeres - en una travesía confortable, posteriormente en otros 30 minutos enlazó Cozumel en otro recorrido sobre el inigualable Caribe y al retornar los satisfechos pasajeros, el capitán Alonso realizó el viaje directo de Cozumel a esta ciudad en una hora y cinco minutos...".

La conectividad que detonó Aerosafari entre Mérida y los destinos del Caribe quintanarroense permitió, en 1965, enlazar Mérida y dos destinos insulares en un tiempo máximo de dos horas y treinta minutos (Rosado, 1981 p.23). En contraste, estudios recientes señalan los conflictos del espacio turístico de la península de Yucatán, caracterizados principalmente por las deficiencias de conectividad entre los destinos insulares como Cozumel (Herrera y Palafox, 2007).

En noviembre de 1966 se anunció que Aerosafari establecería una nueva ruta de nombre "Aeromaya", la cual efectuó un vuelo de Mérida a la ciudad de México (Diario del Sureste, 1-11-1966). Por tanto, la compañía de aviación Aeromaya nació al integrar en 1966 a Aerosafari y a la empresa Servicios Aéreos Gómez Méndez. Con la primera operaba el sureste hasta la Ciudad de México y Acapulco, y con la empresa Servicios Aéreos Gómez Méndez cubría la ruta que iba desde la ciudad de México hacia el Pacífico (Camposeco, 1997).

A partir de 1966 Aeromaya generó un importante movimiento de turismo europeo hacia Yucatán. Como parte de las actividades de publicidad de Barbachano's Travel Service se realizaron campañas en diversos destinos europeos como Londres con la finalidad de promover viajes por la ruta Aeromaya «para conocer la zonas arqueológicas de Yucatán y los bellísimos paisajes del Caribe» (Novedades de Yucatán, 12-11-1966). Desde la perspectiva de los estudios turísticos, la estrategia de Barbachano

7. Diario del Sureste, 1-11-1965. 
Travel Service fue la creación de una cadena de valor de turismo que conjuntara transporte, alojamiento y atracciones turísticas (Vargas, 2013). De este modo, al eslabonar los servicios que prestaba la aerolínea Aeromaya, los hoteles de su propiedad (Mayaland) y la infraestructura y rutas turísticas diseñadas por su agencia de viajes, se generó para esos años lo que hoy se denomina una cadena de valor turístico competitiva.

La solidez de Aeromaya como empresa se evidenció en noviembre de 1966 cuando estalló la huelga entre la Asociación Sindical de Pilotos Aviadores (ASPA) contra la Compañía Mexicana de Aviación (CMA) pues fue la única empresa que continuó prestando servicios ininterrumpidos desde la ciudad de Mérida. Si bien la huelga fue contra la CMA, la situación afectó a empresas como Pan Am, Aerolíneas Vega S.A. y Servicios Aéreos Especiales S.A. (SAESA) que tenían servicios contratados con la CMA como el abastecimiento de combustible. Aeromaya no se vio afectada porque contaba con su propio personal, equipo y sistema de abastecimiento de combustible.

En 1967 iniciaron los problemas. El 2 de junio en la Asociación Sindical de Pilotos Aviadores (ASPA) estalló una huelga contra la empresa Aeromaya. La ASPA sostuvo que en Aeromaya no existía una jornada diaria, ni prestaciones a sus empleados y tampoco un descanso mínimo (Camposeco, 1997). Por su parte, Barbachano Gómez Rul señaló a sus empleados que estas acciones tenían como trasfondo la intención de la Compañía Mexicana de Aviación de acabar con Aeromaya. (Camposeco, 1997). En Mérida, cuando la noticia de la huelga se conoció, se gestó un movimiento en apoyo a los trabajadores de Aeromaya y en contra de Mexicana de Aviación. Se unieron dueños de comercios y restaurantes, al igual que estudiantes universitarios y mostraron su inconformidad con protestas (Pérez, 2007). Después de 102 días, la huelga terminó con la firma del contrato colectivo. Según el Diario de Yucatán (9-07-1967) durante esos días los aviones privados de Aerotaxis brindaron parte del servicio aéreo que Aeromaya había comprometido, con la finalidad de mantener el prestigio de la empresa.

El año de 1968 fue emblemático para México. En octubre la ciudad de México, sede de los Juegos Olímpicos, estuvo convulsionada por los acontecimientos políticos ocurridos diez días antes de la inauguración del evento ${ }^{8}$. No obstante, el evento transcurrió con éxito y atrajo a visitantes de todo el mundo y la llegada de turistas a la ciudad entre septiembre y octubre se incrementó un 37\% respecto el año anterior- (COI, Official Report, 1969). En Yucatán la publicidad de Aeromaya incluyó el tema olímpico y hasta los primeros días de octubre las operaciones de la aerolínea fueron normales. Sin embargo, el 6 de octubre de 1968 según el Boletín Oficial del Colegio de Pilotos, uno de sus aviones «fue desviado de su ruta mientras volaba entre Cozumel y Mérida, siendo llevado a La Habana por una mujer que se decía ser del partido comunista y huía de la persecución del gobierno por su participación en los eventos del 2 de octubre en Tlatelolco ${ }^{9} \gg$. La información al respecto es escasa, se sabe que el aparente secuestro no tuvo víctimas y que el avión aterrizó en el aeropuerto de La Habana.

Aeromaya se sobrepuso a la mala prensa que generó el incidente pero sus días en el aire estaban contados. El 13 de mayo de 1969 Aeromaya cerró definitivamente todas sus operaciones aéreas. La versión oficial difundida por la prensa local señala que a la empresa le fue revocada la concesión debido a irregularidades en la prestación de sus servicios. De este modo, la Secretaría de Comunicaciones y Transportes y el organismo público descentralizado de Aeropuertos y Servicios Auxiliares «ASA» revocaron la concesión de Aeromaya ${ }^{10}$. De inmediato, las rutas aéreas que ofrecía la empresa fueron entregadas por la Secretaría de Comunicaciones y Transportes a la empresa de Servicios Aéreos Especiales S.A. (SAESA). Por parte de la empresa SAESA comunicaron que «debido a irregularidades en el servicio de Aeromaya les cancelaron el permiso, a razón de lo anterior ellos cubrirán las rutas de México-Villahermosa-ciudad del CarmenCampeche y Mérida [...] igualmente cubrirá los vuelos de Isla Mujeres y Cozumel». (Diario del Sureste, 13-05-1969) Sin embargo eso no sucedió y la empresa SAESA si bien operó algunas de las rutas principales, no fue capaz de cumplir con todas las rutas de Aeromaya.

\footnotetext{
8. Durante 1968 se realizó un movimiento estudiantil marcado por la represión y la ocupación del ejército mexicano de las instalaciones de la UNAM y el IPN. Las protestas y las facciones se unieron en una marcha y mitin el 2 de octubre en la plaza de Tlatelolco que finalizó con el ataque del ejército a los estudiantes. Para un análisis 1958-1982 véase Aboites, 2009. 9. [http://colegiodepilotos.org.mx/cabina/bolet48/files/search/searchtext.xml.] 10. Diario Oficial de la Federación, Ley del 12 de junio de 1965.
} 
Las versiones respecto al retiro de la concesión aérea a la empresa yucateca son diversas. El Diario de Yucatán (14-05-1969) menciona que el origen del problema era la falta de manuales de adiestramiento de los pilotos y mecánicos, así como el endeudamiento con la empresa Hawker Sidderley de Inglaterra. Una segunda versión señala que la empresa incumplió el pago de permisos de operación. Una tercera versión explica que la empresa operaba con utilidades, ofrecía costos más bajos en sus rutas y por tanto desplazó en el mercado a sus competidoras de mayor tamaño, que presionaron al gobierno federal para iniciar una campaña que mermara las capacidades de Aeromaya ${ }^{11}$. Un dato importante para entender los procesos posteriores es que desde 1967 la aerolínea Mexicana de Aviación enfrentó serios problemas económicos. En 1968 entró en suspensión de pagos y fue adquirida por un grupo empresarial comandado por el mexicano Crescencio Ballesteros. A partir de ese año inició una reestructuración y según sus registros para 1970 operaba con utilidades y transportó un total de 1,28 millones de pasajeros ${ }^{12}$.

La mala situación económica de Mexicana de Aviación en 1968 convergió con un fenómeno político estatal que buscó el control de las empresas paraestatales. La aviación resultó un sector estratégico para gobierno federal, quien desde 1965 decretó la creación del Organismo Público Descentralizado de Aeropuerto y Servicio Auxiliares «ASA», cuyos objetivos entraron en conflicto con las aerolíneas más pequeñas, como Aeromaya pues surgieron un conjunto de normas para permitir el usufructo de los servicios auxiliares que prestaban las líneas aéreas. La finalidad fue regular los aspectos centrales del transporte aéreo como el abastecimiento de combustible, navegación aérea, aeropuertos, servicio de radar viajes de carga y paquetería ${ }^{13}$. Esta nueva organización no engranó con empresas como Aeromaya que contaban con la infraestructura, el equipamiento y la logística para realizar todas esas actividades de forma independiente.

Con la desaparición de Aeromaya en 1969 concluyó un capítulo central del turismo y la conectividad aérea de la península de Yucatán, marcado

11. [http://manogarci46.wordpress.com/category/sin-categoria/page/155/].

12. [http://mx.reuters.com/article/topNews/idMXN2710225620100827?pa geNumber $=3 \&$ virtualBrandChannel $=0 \&$ sp=true $]$.

13. Diario Oficial de la Federación 12 de junio de 1965 por la operatividad de una cadena de valor turístico exitosa y altamente competitiva. En las décadas posteriores se formaron otras empresas aéreas que operaron con éxito destinos peninsulares, como es el caso de Magnicharters y Aerocaribe, pero no constituyeron un sistema de transporte ni lograron eslabonar una cadena de valor.

\section{Conclusión}

Si bien entre 1931 y 1969 no existen estadísticas oficiales que permitan conocer con exactitud el incremento de llegada de turistas, la consulta de documentos de la época aportan datos que muestran el inicio y rápido crecimiento de la actividad turística en la península de Yucatán. Las evidencias indican la aparición gradual pero imparable de hoteles y otros tipos de hospedaje de menor categoría en los destinos de sol y mar. Así, en Cozumel en 1956 se inauguró el hotel Caribe y comenzó la construcción de las Cabañas del Caribe. En 1961 abrió sus puertas el hotel Martín, en 1962 el Playa Azul, en 1963 los hoteles Candela y López. Un año después, en 1964 comenzaron a funcionar los hoteles Cozumel Caribe y Yoli, en 1966 el Cantarell y el Coldwell. En 1969 se inauguró el hotel El Dorado, los cuartos amueblados Capitán Candela y el magnífico Hotel Presidente. Entre 1970 y 1971 surgen 5 hoteles más, Mara, Malibú, Flores, Pirata y Aguilar. En suma, en los quince años comprendidos entre 1956 y 1971 en un solo destino surgieron 18 hoteles con capacidades instaladas diversas, que iban desde los más pequeños con 10 habitaciones hasta 50 y 80 habitaciones como fue el caso del Playa Azul y el Presidente (Romero, 2014).

Isla Mujeres también fue testigo del crecimiento de la actividad turística. Hacia 1970 Cozumel e Isla Mujeres recibieron 70000 visitantes, la mayoría de ellos extranjeros. En Mérida el auge turístico se vivió de forma distinta. Como el centro de llegadas de los vuelos y el eje que organizaba las rutas al caribe yucateco, fue notorio el surgimiento de desarrollos arquitectónicos que dieran mayor notoriedad histórica y comercial a la ciudad. En 1945 se puso la primera piedra del Monumento a la Patria en el emblemático Paseo de Montejo. En 1950 se realizó la prolongación del Paseo, con la finalidad de crear mayores espacios públicos. El primer hotel ubicado en el Paseo de Montejo se construyó en 1970 (Cámara, 2001). 
Con el desarrollo de la aviación durante los últimos años de la década de los 60's, el crecimiento de la actividad turística en el Caribe Mexicano fue tan evidente que el gobierno federal planificó la construcción del primer complejo turístico de gran escala en el país. La construcción de Cancún inició en 1970 y el primer hotel se inauguró en septiembre de 1974 (Careaga e Higuera, 2010). El surgimiento de Cancún y posteriormente de la Riviera Maya puede considerarse un parteaguas de la historia turística de México (Romero, 2014, Macías y Pérez, 2009). Los fenómenos derivados del desmesurado crecimiento turístico y poblacional en esta área han sido origen de un conjunto significativo de estudios nacionales e internacionales (Marie dit Chirot, 2012, 2014; Balam, 2010). El Caribe Mexicano actualmente es el destino de sol y mar más importante del país y se calcula que en el año 2016 registró 6.2 millones de llegadas de turistas, de los cuales la mayoría arribó por la vía aérea. En 1988, 70 años después de que The Carnegie Institution difundiera el Área Maya, surgió el Programa Mundo Maya, una iniciativa de la Organización Mundial del Turismo (OMT) y la Comunidad Económica Europea con el apoyo de los gobiernos de los 5 países con asentamientos mayas (Belice, Honduras, Guatemala, El Salvador y México). En el 2011 el Programa Mundo Maya reanudó reuniones y reactivó sus planes de trabajo con miras al auge turístico esperado para el 2012. Los resultados fueron desiguales en los cinco paises participantes y la integración de una ruta turística internacional es aún una expectativa que no se cumple. El problema principal es la conectividad aérea ${ }^{14}$.

\section{Bibliografía}

Aвortes L., 2009, El último tramo, 1929-2000, en Nueva Historia Mínima de México, México, El Colegio de México, $315 \mathrm{p}$.

Balam, Y., 2010, Tulum: Mayas y Turismo, México, Universidad de Quintana Roo, 379 p.

Barrera A., En busca de los antiguos mayas. Historia de la Arqueología en Yucatán, Mérida, Editorial Dante, 104 p.

Boyer M., 2002, El Turismo en Europa, de la Edad Moderna al siglo XX, Historia Contemporánea 25, p. 13-31.

Buergo J., Morlett F., Nacimiento de la aviación comercial mexicana, [www.mexicanaviationhistory.com].

14. El Economista, 13 de marzo 2013; 17 de febrero 2015.
Camposeco, V., 1977, Ciento dos días de huelga, diez años después, Revista Hélice no 194, Año XVIII, mayo, p. 16-19.

Careaga L., Higuera A., 2010, Quintana Roo, Historia Breve, México, El Colegio de México, Fondo de Cultura Económica, 287 p.

Carranza E., 1976, Resumen Histórico de la Aeronavegación. Del globo Montgolfier al supersónico Concorde de nuestros días, México D.F., Costa-Amic Editor, 108 p.

Coltman M., 1998, Introduction to Travel and Tourism: An International Approach, Editorial Van Nostrand Beihold, $384 \mathrm{p}$.

Comité Olímpico Internacional, 1969, Official Report of the Organizing Committee of the Games of the XIX Olympiad, Tomo II, p. 167.

García B., 2006, Las regiones de México. Breviario geográfico e histórico, México D.F., El Colegio de México, 351 p.

García de Fuentes, A., Jouault, S., Romero D., 2015, Atlas de Turismo Alternativo de la Península de Yucatán, Cinvestav, Mérida, México, 170 p.

García de Fuentes A., Xool M., 2012 Turismo Alternativo y Desarrollo en la Costa de Yucatán, Mérida, México, Cinvestav, $196 \mathrm{p}$.

Hernández E., 2005, De hélice al Jet de largo alcance. La Compañía Mexicana de Aviación 1960-1990, Memorias del II Congreso AMHE. [http://www.amhe/memoria/simposio 13/ Erik HERNANDEZ.pdf].

Historia de los caminos de México, 1994, Tomo 3-Siglo XX, Banco Nacional de Obras y Servicios Públicos SNC, México, 280 p.

Izquierdo R. (ed.), 1994, Transportes, un enfoque integral, Tomo I, Colegio de Ingenieros de caminos, canales y puertos, España, 332 p.

Jiménez A., 1993, Turismo: estructura y desarrollo, México, Ed. McGraw Hill, 487 p.

Lapointe M., 2008, Historia de Yucatán, Siglos XIX-XXI, Universidad Autónoma de Yucatán, Mérida, 316 p.

López Pardo G., Palomino B., 2007, Elaboración de un diagnostico del estado de la investigación turística en el país y generación de un programa de investigación integral para el sector turismo. Universidad Nacional Autónoma de México, Instituto de Investigaciones Económicas, CESTUR.

Macías C., Pérez R., 2009, Cancún, los avatares de una marca turística global, Universidad de Quintana Roo, México, Ed. Bonilla Artigas, 476 p.

Marie dit Chirot C., 2012, La ville négociée. Flux et reflux dans une mobilisation locale pour l'accès au logement à Playa del Carmen, Mexique, Géocarrefour, Vol. 87/1, p. $37-46$.

Marie dit Chirot C., 2015, Relaciones de propiedad y conflictos por la apropiación del espacio turístico. Análisis comparativo entre Huatulco (Oaxaca) y Playa del Carmen (Quintana Roo) en México, en Marín G. (dir.), Sin tierras no hay paraíso. Turismo, organizaciones agrarias y apropiación territorial en México, Revista Pasos, p. 251-274.

Muñoz F., 2004, Autopsia del Turismo: El vencimiento de la distancia, Oviedo, LibrosenRed, 144 p. 
Olsen J., 2010, A History of Air Warfare, Potomac Books, Virginia, $522 \mathrm{p}$.

Page S., Connell J., 2006, Tourism: A Modern Synthesis, Cengage Learning, Emea, 515 p.

Palacios G., 2012, Los Bostonians, Yucatán y los primeros rumbos de la arqueología americanista estadounidense, 18751894. Historia Mexicana 62, n 1, julio-sept., p. 105-193.

Palacios G., 2015, El cónsul Thompson, los Bostonians y la formación de la galaxia Chichén, 1893-1904. Historia Mexicana $65, n^{\circ} 1$, julio-sept., p. 167-288.

PARÉ, L., FrAGA J., 1994, La costa de Yucatán: desarrollo y vulnerabilidad ambiental. Cuadernos de Investigación $\mathrm{N}^{\circ} 23$, Instituto de Investigaciones Sociales, México.

Pool M., 2014, Las reinvenciones de la etnicidad en la arqueología maya, Universidad Autónoma de Yucatán, 299 p.

Pérez J., 2007, Las luchas estudiantiles en México, 1901-1980, México, Secretaría de Prensa y Propaganda del STUNAM.

[http://www.stunam.org.mx/8prensa/cuadernillos/cuaderno90. html].

Pérez de Sarmiento M., Savarino F., 2001, El cultivo de las élites. Grupos económicos y políticos en Yucatán en los siglos XIX y XX, Consejo Nacional para la Cultura y las Artes, $261 \mathrm{p}$.

Quezada S., 2001, Breve Historia de Yucatán, México D.F., El Colegio de México, Fondo de Cultura Económica, 288 p.

Rico A., 2001, El transporte aéreo de carga doméstica en México, Publicación Técnica no. 168, Secretaría de Comunicaciones y Transportes, Querétaro, 157 p.
Ricketson O., Kinder A., 1930, An Archeological Reconnaissance by Air in Central America, The Geographical Review, vol. XX, n ${ }^{\circ}$, abril, American Geographical Society of New York, p. 177-206.

Romero R., 2014, Turismo en el caribe mexicano, México, Bonilla Artigas, Universidad de Querétaro, 237 p.

Ruiz M., 2011, 100 años de la aviación en México, Tomo I, México, Secretaría de Comunicaciones y Transportes, $325 \mathrm{p}$.

Rosado R., 1979, Vuelo al pasado. Yucatán en las proezas iniciales de la aviación mexicana, Mérida, Komesa, 96 p.

Rosado R., 1981, Resumen histórico de la aviación en Yucatán, Mérida, 46 p.

The Economist, 2010, Mexican Airlines: A clumsy giant stumbles, 14 de agosto [http://www.economist.com/ node/16797757].

Transporte y Logística, 2006, Crónica de Legislación y Jurisprudencia, Revista Actualidad Jurídica Uría Menéndez, $\mathrm{n}^{\circ}$ 13, p. 205-211.

Vargas L., 2013, Desarrollo logístico del transporte en el Quindío. Una opción de valor para el turismo, Global Conference on Business and Finance Proceeding, Vol. 8, num. 2, p. 1082-1089. 